\title{
Prospective Randomized Study for Antibiotic Prophylaxis in Spine Surgery: Choice of Drug, Dosage, and Timing
}

\author{
Nishant ${ }^{1}$, Kannan Karthick Kailash ${ }^{2}$, P.V. Vijayraghavan ${ }^{2}$ \\ ${ }^{1}$ Orthopedic Surgeon and Spine Surgeon, Rameshwaram Orthopedic and Spine Clinic, Patna, India \\ ${ }^{2}$ Department of Orthopaedic Surgery, Sri Ramachandra Medical College and Research Centre, Chennai, India
}

\begin{abstract}
Study Design: Prospective randomized study of antibiotic prophylaxis in elective spine surgery.
Purpose: The aim of this study was to compare the rate of postoperative surgical site infection for a single dose of two different generations of cephalosporin with different dosage and timing of the antibiotics.

Overview of Literature: Current recommendation for prophylaxis in elective spine surgery is up to 60 minutes prior to incision. No study has investigated between different generation of cephalosporin for prophylaxis in elective spine surgery with respect to choice, dosage and timing.

Methods: This study was a prospective randomized study of 90 patients, assessed for the occurrence of surgical site infection (defined by the Centers for Disease Control and Prevention criteria) and other infections for up to 6 months after surgery. Demographic, surgical and further data were collected on subsequent operations, including hardware removal.

Results: Mean age in our group was 47 years (range, 19-71 years). The male to female ratio was $49: 41$ and the average timing of administration of antibiotics was 77 minutes (range, 30-120 minutes). The average blood loss was $626 \mathrm{~mL}$ (range, 150-3,000 mL) with a mean duration of surgery for 3.2 hours (range, 1.5-6 hours). One case of superficial infection and one case of deep infection met the exclusion criteria.

Conclusions: Our results support the use of a single preoperative dose of antibiotics in instrumented and non-instrumented elective spine surgery up to one hour prior to incision. There was no difference in terms of occurrence of surgical site infection with respect to dosage, choice and timing of antibiotics.
\end{abstract}

Keywords: Antibiotic prophylaxis; Spine; Surgical site infection

\section{Introduction}

Despite advances in prophylaxis, the incidence of deep infection after instrumented spinal surgery remains between $0.4 \%$ and $10 \%$ [1]. For the recent evidence-based clinical guideline for the use of prophylactic antibiotics in spine surgery, eleven clinical questions addressed the efficacy and appropriateness of antibiotic prophylaxis protocols, repeat dosing, discontinuation, wound drains and special considerations related to the potential impact of comorbidities on antibiotic prophylaxis [2]. The respective recommendations were graded by the strength

Received Sep 5, 2012; Revised Sep 27, 2012; Accepted Sep 27, 2012

Corresponding author: Nishant

Orthopedic Surgeon and Spine Surgeon, Rameshwaram Orthopedic and Spine Clinic,

K-79, P.C.Colony, Hanuman Nagar, Kankarbagh Colony, Patna-800020 (Bihar), India

Tel: +91-9525517801, Fax: +91-612-235137, E-mail: nishantspine@gmail.com 
of the supported literature, which was stratified by levels of evidence in this published work by Watters et al. [2]. However, there were many answered questions with respect to the appropriate dosage timing and superiority of one drug regime over another. It is a known fact that instrumentation increases the risk of infection. Appropriate timing for various Orthopaedic procedures has been documented. However, the intervertebral disc is relatively limited in blood supply, which leads to less penetration and drug delivery across this region $[3,4]$. Numerous randomized studies have been performed for timing and dosage of the antibiotic prophylaxis [5,6]. A recent metaanalysis of six open, prospective, nonrandomized trials or subgroup suggested that prophylactic antibiotics were beneficial for spinal surgery, even if the infection rates, in the absence of antimicrobial prophylaxis, favor antibiotic prophylaxis [6]. However, meta-analyses are not exempt from the bias. In a number of areas of clinical controversy in which nonrandomized trials were unable to yield a clinical consensus, randomized clinical trials produced clear results while exposing far fewer patients to ineffective therapy [7]. Moreover, the above-mentioned metaanalysis did not distinguish superficial and deep surgical site infections, particularly spondylodiscitis, the burden of which is substantially different. The previous study did not prove that cefuroxime is better than other antibiotics frequently used for prophylaxis, such as first generation cephalosporins [8].

\section{Materials and Methods}

This prospective randomized study was performed in our Department of Orthopaedic Surgery at Sri Ramachandra University (SRU), Porur, Tamil Nadu, India. The study protocol was approved by the ethical committee at SRU University and informed written consent was obtained from each patient. Patients older than 18 years requiring spinal surgery were recruited from 1st May 2010 through 31st May 2011. The exclusion criteria were as follows: known or suspected hypersensitivity to cephalosporins or type I hypersensitivity to betalactams; severe renal function impairment; acquired immune deficiency syndrome or other conditions of severe immunosuppression; antibiotic therapy for concomitant infection at the time of surgery; refusal to participate; or pregnancy. The patients were randomly assigned into three different groups and allocated to receive one vial of administration of preoperative antibiotics with group I-1 g cefazolin (first generation cephalosporin), group II-1 g cefuroxime (second generation cephalosporin), and group III-1.5 g cefuroxime, respectively. Preoperative antibiotic were given in both instrumented and non-instrumented spine surgery as per institution policy. Postoperative dose was continued in both instrumented and non-instrumented spine surgery for 48 hours [9]. All cases were operated by a single spine surgeon. All cases received the same antibiotics preoperatively (single dose) and postoperatively for 48 hours [9]. The first intraoperative dose was additionally given if the surgery exceeded 2 hours or if there were more than three comorbid factors $[10,11]$. The second intraoperative dose was given if blood loss was greater than $1,500 \mathrm{~mL}$ or if the duration of surgery was more than 6 hours from skin incision (4 hours from the first dose). However, postoperatively, patients were not blinded because the operating surgeon was aware of the type of antibiotics given later in the study, when the coded was decoded. Choice of antibiotic was given randomly by a trained nurse under the supervision of a senior anesthetist not involved in the study. Drain was removed on the second day if the drainage was less than $75 \mathrm{~mL}$. Wound inspection and dressing were done as per hospital established protocols on the second, fifth and 11th postoperative day. Suture removal was performed on the 11th postoperative day. An orthopedic resident not involved in the study collected the data.

Randomization of the drug was performed by the hospital pharmacist according to a computer-generated random scheme [11]. Numbered vial of identical appearance containing $1 \mathrm{~g}$ of cephazolin and $1.5 \mathrm{~g}$ of cefuroxime are allocated to the patients and administered on arrival at the operating theatre, approximately 2 hours before surgery. The pharmacist was informed about the use of implants in that surgery. In case the patient needed an implant, the pharmacist decides to randomly give either of the three $(1 \mathrm{~g}$ cefazolin, cephazolin vs. $1 \mathrm{~g}$ and $1.5 \mathrm{~g}$ cefuroxime) antibiotics. All three groups which received antibiotics were prepared and numbered by the hospital pharmacies, and were administered intravenously.

Preoperative randomization of the timing of administration: all three groups which received drugs by the head nurse, administered preoperatively at 2 hours (120 minutes) before surgery, were divided into 4 groups; group I, $<30$ minutes; group II, 31 to 60 minutes; group III, 61 to 90 minutes, and group IV, 91 to 120 minutes. The nurs- 
ing staff was also unaware of which drug was given. The timing of administration was supervised by the anesthesiologist at the time of induction and was documented on the anesthesiology sheet. The surgeon was asked about the occurrence of a break in asepsis during operation. Patients were observed prospectively during hospitalization by a resident in orthopedics in case of any abnormal findings. The following clinical information was recorded: temperature, symptoms or signs of infection, diagnostic tests performed, prescription of antibiotics, and analgesics or anti-inflammatory drugs. All patients had urine cultures before surgery. The patients were mobilized 24 hours after surgery, and have leg and breathing exercises. The urinary catheter was removed on the second postoperative day. Six weeks after the operation, the wound was examined by an independent observer who is unaware of the prophylactic regime. During this follow-up visit, a standardized questionnaire recorded information about the difficulties in wound healing, evidence of any infection during the follow-up, unscheduled visits to a physician and prescription of antibiotics [11]. All patients are also seen in person at 6 weeks, 3 months and 6 months after the operation in order to collect standardized follow-up data.

\section{Outcome measures}

Surgical site infection (SSI) is defined according to the criteria of the Centers for Disease Control and Prevention (CDC) [12]. A superficial infection of the incision site is diagnosed in the presence of clinical signs of infection that involves the skin, subcutaneous tissue, or muscle located above the fascial layer, and accompanied by at least one of the following criteria: purulent drainage located above the fascial layer and/or isolation of a microorganism from a wound culture showing signs of inflammation. A deep incisional infection is diagnosed when the infection is found to involve tissue below the fascia on opening and exploration by the surgeon. An organ space infection is diagnosed when clinical and imaging (CT scan or magnetic resonance imaging) signs are suggestive of an infection of a disc, a vertebra, or a paravertebral structure, and is associated or not associated with a positive culture of blood or of the material obtained by a needle aspiration of the vertebral space, needle biopsy of the bone, or open bone biopsy. Deep SSI are required to meet the following criteria: 1) the infection occurred within 30 days after the operative procedure if no implant was left in place and within 1 year if implant was in place; 2 ) it seemed to be related to the operative procedure; 3 ) it involved the deep soft tissue of the incision (fascial and muscle layer) or an organ space that was opened or manipulated during the operative procedure; and 4) the patient had either (a) purulent drainage from the deep incision; (b) deep incision spontaneously dehisced or was opened by a surgeon; (c) evidence of infection involving the deep tissue on direct examination; or (d) diagnosis of deep infection by a surgeon [12].

Postoperative infections other than surgical site infections were diagnosed according to the CDC criteria $[12,13]$, which included a diagnosis of infection by the surgeon. All included patients are monitored for signs of drug toxicity and serious adverse events, such as Clostridium difficile associated colitis, allergic reaction or anaphylactic shock. Demographic and surgical data collected included the age at the time of surgery, gender, underlying medical conditions, history of previous spinal surgery and other surgeries, dosing and timing of preoperative antibiotic prophylaxis, diagnosis, type of surgery, use of instrumentation, use of allograft, number of vertebrae fused, estimated blood loss, and duration of surgery. For patients with an occurrence of surgical site infection, further data were collected on subsequent operations, including hardware removal. Postoperative data included symptoms and signs of infection with a follow-up of at least 6 months. Infections were determined by positive wound culture and sensitivity or by an attending spine surgeon's clinical impression that wound infection had occurred.

\section{Statistical analysis}

The mean age in our group was 47 years (range, 19-71 years). The male to female ratio was 49:41 and the average timing of administration of antibiotics was $77 \mathrm{~min}$ utes (range, 30-120 minutes). The average blood loss was $626 \mathrm{~mL}$ (range, 150-3,000 mL) with a mean duration of surgery for 3.2 hours (range, 1.5-6 hours). One case of superficial infection and one case of deep SSI was noted. Complex statistical analysis was avoided because for a wound infection rate of $10 \%, 5 \%$ and $2 \%$, it would be necessary to enroll 474, 988, and 2,518 subjects, respectively, in each treatment group [14], 5,036 patients would need to be enrolled to show a decrease in the infection rate to 
Table 1. Demographic and surgical data among the three groups

\begin{tabular}{|c|c|c|c|}
\hline & $1 \mathrm{~g}$ Cefazolin & $1 \mathrm{~g}$ Cefuroxime & $1.5 \mathrm{~g}$ Cefuroxime \\
\hline Male:female & 18:15 & $17: 13$ & $18: 9$ \\
\hline \multicolumn{4}{|l|}{ Median age in year (range) } \\
\hline Male & $21-65$ & $30-71$ & $21-68$ \\
\hline Female & $19-69$ & $27-64$ & $23-61$ \\
\hline Instrumentation & I-25; NI-8 & I-26; NI-3 & I-16; NI-8 \\
\hline PSDF & 5 & 1 & 7 \\
\hline PSDFI & 10 & 21 & 8 \\
\hline Fenestrectomy and diskectomy & 1 & 0 & 0 \\
\hline ACDFI & 0 & 1 & - \\
\hline Deformity correction+IF & 2 & 2 & 1 \\
\hline P.V & 2 & 1 & 1 \\
\hline Stage 2 ant & 0 & 1 & 0 \\
\hline \multicolumn{4}{|l|}{ Interbody fusion } \\
\hline Revision PSDI & 0 & 1 & 0 \\
\hline Revision PSDFI & - & 2 & - \\
\hline PSS+IF & 1 & 1 & 2 \\
\hline PSS+BG+pars repair & 1 & 0 & 0 \\
\hline $\mathrm{PSS}+\mathrm{V}$ & 1 & 0 & 0 \\
\hline PSS+pars repair & 1 & 0 & 0 \\
\hline PSS & 8 & 1 & 2 \\
\hline PSDI & 1 & 0 & 0 \\
\hline Allograft & 8 & 10 & 4 \\
\hline$>1$ Levels fused & 11 & 15 & 7 \\
\hline
\end{tabular}

PSDF, posterior spinal decompression and fusion; PSDFI, posterior spinal decompression, fusion, and instrumentation; ACDFI, anterior corpectomy, decompression, fusion, and instrumentation; IF, instrumented fusion; P.V, percutaneous vertebroplasty; PSS, posterior spinal stabilization; BG, bone grafting; V, vertebroplasty; PSDI, posterior spinal decompression and instrumentation.

1\%. Such large sample size establishes that a clinical trial is unlikely to occur in which the infection rate is as low as $2 \%[6,13]$.

\section{Results}

Out of the 90 patients, we report only one case of superficial infection and one case of deep infection which met the exclusion criteria. The patient with superficial infection was diagnosed with intervertebral disc prolapse L4L5 level with lumbar canal stenosis, for which posterior spinal decompression and fusion were performed. The patient was given $1.5 \mathrm{~g}$ of cefuroxime; further, no break sepsis was noted during the intraoperative period. The patient developed a wound discharge, which was noted on the fifth postoperative day during wound inspection and dressing. The wound culture was taken and showed staphylococcus aureus sensitive to ciprofloxacin. The patient was treated with oral antibiotics for 5 days. During the follow-up, no further signs of infection were documented. No adverse reaction of prophylaxis was noted in any patient. Tables 1, 2 illustrates the surgical and demographic data in each group.

\section{Discussion}

Antimicrobial prophylaxis is effective, if the bactericidal activity against the most common infecting organisms is present in the tissues at risk from the time of the incision for the duration of the procedure as long as the serum concentration is maintained. The amount of blood loss of more than $1,500 \mathrm{~mL}$ as well as the duration of surgery 
Table 2. Choice, dosage and timing of antibiotic administration in each group

\begin{tabular}{|c|c|c|c|}
\hline Choice of antibiotic & $1 \mathrm{~g}$ Cefazolin & $1 \mathrm{~g}$ Cefuroxime & $1.5 \mathrm{~g}$ Cefuroxime \\
\hline No. of patients & 33 & 30 & 27 \\
\hline Average time (min) & 75 & 82 & 77 \\
\hline \multicolumn{4}{|l|}{ Min of dosing } \\
\hline Type of surgery & I-25; NI-8 & I-26; NI-3 & I-16; NI-8 \\
\hline Blood loss (mL) & 490 & 565 & 658 \\
\hline Duration of surgery (hr) & 2.9 & 3.2 & 3.4 \\
\hline Wound infection & - & $1 \mathrm{D}$ (satisfied exclusion criteria) & 1 Superficial infection \\
\hline
\end{tabular}

No cases had more than 3 morbidity risk factors in any group $[10,11]$.

I, stands for instrumented surgery; NI, non-instrumented surgery; 1 D, 1 case of deep infection.

can affect the clearance of the antibiotic. Based on these principles, the most efficacious and judicious use of antibiotics can be achieved. Antibiotic prophylaxis significantly reduces the incidence of postoperative superficial and deep infection [6,13-16]. Elective spinal surgical procedures fall into the category of clean surgery, with a low rate (about 2\%) of postoperative infections [6,13], except for iatrogenic discitis for which the incidence ranges from $1 \%$ to $5 \%[17,18]$. This needs special mention because it accounts for one the most common cause for failed back surgery. The ideal choice of antibiotics for prophylaxis of wound infection should have limited toxicity, relative low cost and a broad coverage of organisms; however, it should be limited to avoid resistance and super infection.

\section{Choice of antibiotic and dosing}

Current practice for the choice of antibiotic is either a first or second generation cephalosporin, based on the fact that they have better coverage against gram positive organisms which are the most common cause for spine infection. Rubinstein et al. [19] conducted a double-blind, placebo controlled trial and recommended an administration of a single dose of cephazolin preoperatively for patients undergoing lumbar spinal surgery, provided that sensitivity monitoring is performed in all infected cases. An earlier animal study [20] concluded $1 \mathrm{~g}$ of intravenous cephazolin to be highly effective for prophylaxis against bacterial contamination [19-22]. In contradiction to this, a study by Walters et al. [3] concluded that the incidence of iatrogenic discitis cannot be eliminated with a broad spectrum antibiotic, such as cephazolin. Moreover, cephazolin appeared to be ineffective at preventing endplate destruction once discitis has become established. Current evidence exists to suggest that the charge on antibiotics, due to their ionisable groups, is important in determining their ability to diffuse into the disc [23]. Variation in the rate of diffusion for different antibiotics may be due to multiple factors, including molecular size and charge, serum protein binding, and antibiotic solubility in different tissues. Rhoten et al. [22] and Lang et al. [24] suggested that higher doses of cephalosporins are required to achieve therapeutic levels in the disc. Hoelscher et al. [25] have shown that high doses of antibiotics, including both cephalosporins and aminoglycosides, could have deleterious effects on the survival of cultured disc cells, cell proliferation and metabolic rates. In Gibson et al. [26], series, negatively-charged antibiotics were not detected in either the nucleus pulposus or the annulus fibrosus of a normal human intervertebral disc, despite high blood levels of antibiotics. It is therefore likely that the penetration of antibiotics is similar in normal and pathological discs. Therefore, the literature has conflicting evidence regarding the concept of higher dosing as well as the charge on the antibiotic as the determinant factor. Besides, it is notable that both cefazolin and cefuroxime are positively charged ions, but with a longer half-life of cefazolin. In a double-blind, placebo controlled clinical study by Petignat et al. [5], a single, preoperative dose of $1.5 \mathrm{~g}$ cefuroxime reduces the risk of organ space infection, most notably spondylodiscitis, after surgery for herniated disc in non-instrumented spine surgery. The overall rate of infection is $5.2 \%$ in the cefuroxime group and $6.3 \%$ in the placebo. However, it did not prove that cefuroxime was better than other antibiotics frequently used for prophylaxis, such as first-generation cephalosporin. 
Table 3. Timing of administration of antibiotics and additional doses of antibiotics in each group

\begin{tabular}{|c|c|c|c|c|c|c|c|}
\hline \multirow{2}{*}{$\begin{array}{l}\text { Timing of } \\
\text { antibiotic } \\
\text { (min) }\end{array}$} & \multicolumn{3}{|c|}{ Group } & \multirow{2}{*}{ 1st Intra-op dose } & \multirow{2}{*}{ 2nd Intra-op dose } & \multirow{2}{*}{$\begin{array}{c}\text { Duration of } \\
\text { surgery }\end{array}$} & \multirow{2}{*}{ Blood loss } \\
\hline & I & II & III & & & & \\
\hline 30 & 4 & 1 & 1 & Yes in all patients & $\begin{array}{l}\text { Gp I-1 } \\
\text { Gp II-0 } \\
\text { Gp III-0 }\end{array}$ & 197 & $\begin{array}{l}>1,500 \mathrm{~mL} \mathrm{Gp} \mathrm{I} \\
(>3 \mathrm{~L})\end{array}$ \\
\hline $31-60$ & 10 & 9 & 8 & $\begin{array}{l}\text { Gp I-7 } \\
\text { Gp II-4 } \\
\text { Gp III-6 }\end{array}$ & $\begin{array}{l}\text { Gp I-0 } \\
\text { Gp II-0 } \\
\text { Gp III-1 }\end{array}$ & 193 & $\begin{array}{l}>1,500 \mathrm{~mL}(1.8 \mathrm{~L}) \\
\text { Gp III }\end{array}$ \\
\hline $61-90$ & 13 & 10 & 7 & $\begin{array}{l}\text { Gp I-9 } \\
\text { Gp II-7 } \\
\text { Gp III-6 }\end{array}$ & $\begin{array}{l}\text { Gp I-0 } \\
\text { Gp II-1 } \\
\text { Gp III-0 }\end{array}$ & 195 & $\begin{array}{l}<1,500 \mathrm{~mL} \\
\text { duration }>6 \mathrm{hr} \\
\text { Gp II }\end{array}$ \\
\hline $91-120$ & 6 & 9 & 8 & $\begin{array}{l}\text { Gp I-6 } \\
\text { Gp II-9 } \\
\text { Gp III-8 }\end{array}$ & $\begin{array}{l}\text { Gp I-0 } \\
\text { Gp II-0 } \\
\text { Gp III-3 }\end{array}$ & 194 & $\begin{array}{l}<1,500 \mathrm{~mL} \\
\text { duration>6 hr } \\
\text { Gp III }\end{array}$ \\
\hline
\end{tabular}

Intra-op, intraoperative; Gp, group; Gp I, cefazolin $1 \mathrm{~g}$; Gp II, 1 g cefuroxime; Gp III, $1.5 \mathrm{~g}$ cefuroxime.

\section{Timing of administration}

From our point of view, the timing of administration is the most controversial to establish the exact safer limit for the timing of antibiotics administration. According to Classen et al. [27], the rate of wound infections are lowest if when the antibiotics are administered within 2 hours of surgery. The current opinion [13] is that patients should receive antibiotics 30 minutes prior to the skin incision and preferably before induction. This measure allows time for the antibiotic to reach tissues with optimal bactericidal serum concentration. Operative procedures proceeding more than 6 hours of procedures with instrumentation (implants prosthetic materials), diabetes, obesity, smoke, severe blood loss more than $1,500 \mathrm{~mL}$, remote source of infection, and 3 or more co existing medical comorbids are additional risk factors for postoperative wound infection, thereby warranting redosing of antibiotic prophylaxis [17,18]. Fraser et al. [20] and Guiboux et al. [28], using sheep and rabbit models of discitis, respectively, demonstrated that the administration of intravenous cefazolin prevented the development of infection. These studies [20] demonstrated that the optimal time of administration was 30 to 60 minutes before surgery. Also, they found that the concentration of cefazolin in the disc was dependent on the maintenance of high serum antibiotic concentrations; thus, making it imperative to ensure the proper timing and dose of antibiotics. Once infection develops in the disc, intravenous antibiotics have little impact on the course of the disease.
Some authors have advocated antibiotics 24 hours postoperatively [13]. Other studies have affirmed that additional administration have no value $[27,29]$, even in the presence of wound drain and epidural catheter [30]. In our set up, we gave postoperative antibiotics for 48 hours due to the presence of drains and epidural catheter, as per a recent study by Kim et al. [9]. We do not consider fever alone as a parameter for continuing antibiotic as it is our clinical experience that it is surgical stress induced rather than a marker of infection. It has been our clinical experience that maintaining the therapeutic level is more important. In our series, out of 30 patients for whom antibiotics were given between 61 to 90 minutes, only 8 patients did not have intraoperative dosing as mentioned in Table 3. None of those patients developed an infection. These 8 patients had posterior decompression with instrumentation and fusion in all three different groups of antibiotics. Based on our findings that antibiotics are not detectable between 61 to 90 minutes can be challenged. Interestingly, all patients who received antibiotics beyond 90 minutes had an intraoperative dose (Table 3), given which may even challenge the role of prophylaxis itself and suggest that intraoperative dosing may be a better option. However, we have a very small subset of patients to come to this conclusion. Looking at the average duration among all three groups of antibiotics by data analysis, we came to conclusion that 75 minutes is safer and better in all groups where it is highly unlikely that patients will develop infection. Barker [6] conducted a metanalysis and concluded that antibiotic prophylaxis was beneficial 
for spinal surgery. The rate of infection was $5.9 \%$ in this metanalysis for patients without antibiotics, which was $2 \%$ lower in the control group (2.9\%). The controversy has been that routine antibiotic prophylaxis leads to possible drug reactions, emergence of antibiotic resistance against bacteria and additional cost $[15,16]$. Others argue that prophylaxis for most spinal surgery should be used for internal fixation or fusion of spine as they are associated with higher risk of infection compared to surgeries without instrumentation and extensive surgical exposure. Our clinical observation was that if there is no break in asepsis during surgery, most of the superficial infection are acquired after surgery and reflects the standard of asepsis enforced during postoperative care. However, the data from our present study established that if high standards of asepsis are enforced, rate deep infections can also avoided. Our data reveals that classic microorganisms were recovered from one case of superficial infection. The culture and sensitivity was sent and patients were treated as per sensitivity of the organism. However, the limitation of our study was that we had no case of methicillin resistant Staphylococcus aureus (MRSA) or resistant organisms to cefazolin. We do not have data to support any specific guidelines regarding this particular infection and we still follow the standard practice as for treating any MRSA infected patients in any surgery worldwide. There was no adverse event reported in relation with the use of prophylaxis and is coherent with other studies.

The present study suffers from a few limitations. The present study is a randomized study, which was initially blinded to the nurse, patients and the resident collecting the data. However, the blinding was lost because a single spine surgeon involved in surgery was aware of the postoperative dose and the choice of antibiotic given. Due to the lack of a control group in this study of patients without instrumented surgery, the role of prophylaxis antibiotic may be challenged. However the economic, social and psychological burden far exceeds the consequences, if patients develop any surgical site infection. Another limitation comes from the fact that the diagnosis of SSI relied largely on the physician in charge. The impact of this possible bias is limited though not free because it was not a blinded study. One more limitation of our study regarding deep SSI is that as per CDC, the criteria minimum of a one year follow-up is necessary. Our study has conducted a follow-up only for 6 months. Although the initial results have been very encouraging, a double- blinded multicenter study across the subcontinent would be needed in order to answer other key issues and limitations.

\section{Conclusions}

Our results support the use of a single preoperative dose of antibiotics in instrumented and non-instrumented spine surgery. There was no difference among the first versus second generation antibiotics with respect to dosage ( $1.5 \mathrm{~g}$ vs. $1 \mathrm{~g}$ cefuroxime and $1 \mathrm{~g}$ cefazolin) in occurrence of surgical site infection. We recommend prophylaxis up to 60 minutes prior to incision as previously established. However, we recommend a multicenter double-blind study in the subcontinent to further substantiate our findings and answer the few key remaining questions pertaining to the limitations of this study.

This prospective randomized study demonstrates a single dose of $1 \mathrm{~g}$ of cefazolin, $1.5 \mathrm{~g}$ or $1 \mathrm{~g}$ of cefuroxime before spinal surgery reduces the risk of superficial and deep infection.

\section{Conflict of Interest}

No potential conflict of interest relevant to this article was reported.

\section{References}

1. Sasso RC, Garrido BJ. Postoperative spinal wound infections. J Am Acad Orthop Surg 2008;16:330-7.

2. Watters WC 3rd, Baisden J, Bono CM, et al. Antibiotic prophylaxis in spine surgery: an evidence-based clinical guideline for the use of prophylactic antibiotics in spine surgery. Spine J 2009;9:142-6.

3. Walters R, Rahmat R, Fraser R, Moore R. Preventing and treating discitis: cephazolin penetration in ovine lumbar intervertebral disc. Eur Spine J 2006;15:1397403.

4. Walters R, Rahmat R, Shimamura Y, Fraser R, Moore R. Prophylactic cephazolin to prevent discitis in an ovine model. Spine (Phila Pa 1976) 2006;31:391-6.

5. Petignat C, Francioli P, Harbarth S, et al. Cefuroxime prophylaxis is effective in noninstrumented spine surgery: a double-blind, placebo-controlled study. Spine (Phila Pa 1976) 2008;33:1919-24.

6. Barker FG 2nd. Efficacy of prophylactic antibiotic 
therapy in spinal surgery: a meta-analysis. Neurosurgery 2002;51:391-400.

7. Altman DG. Systematic reviews of evaluations of prognostic variables. In: Egger M, Davey Smith G, Altman DG, editors. Systematic reviews in health care: meta-analysis in context. 2nd ed. London: BMJ Books; 2001. p.228-47.

8. Bratzler DW, Houck PM; Surgical Infection Prevention Guidelines Writers Workgroup, et al. Antimicrobial prophylaxis for surgery: an advisory statement from the National Surgical Infection Prevention Project. Clin Infect Dis 2004;38:1706-15.

9. Kim B, Moon SH, Moon ES, et al. Antibiotic microbial prophylaxis for spinal surgery: comparison between 48 and 72-hour AMP protocols. Asian Spine J 2010;4:71-6.

10. Haley RW, Culver DH, Morgan WM, White JW, Emori TG, Hooton TM. Identifying patients at high risk of surgical wound infection: a simple multivariate index of patient susceptibility and wound contamination. Am J Epidemiol 1985;121:206-15.

11. Larsen RA, Evans RS, Burke JP, Pestotnik SL, Gardner RM, Classen DC. Improved perioperative antibiotic use and reduced surgical wound infections through use of computer decision analysis. Infect Control Hosp Epidemiol 1989;10:316-20.

12. Horan TC, Gaynes RP, Martone WJ, Jarvis WR, Emori TG. CDC definitions of nosocomial surgical site infections, 1992: a modification of CDC definitions of surgical wound infections. Infect Control Hosp Epidemiol 1992;13:606-8.

13. Garner JS, Jarvis WR, Emori TG, Horan TC, Hughes JM. CDC definitions for nosocomial infections, 1988. Am J Infect Control 1988;16:128-40.

14. Dimick JB, Lipsett PA, Kostuik JP. Spine update: antimicrobial prophylaxis in spine surgery: basic principles and recent advances. Spine (Phila Pa 1976) 2000;25:2544-8.

15. Riley LH 3rd, Banovac K, Martinez OV, Eismont FJ. Tissue distribution of antibiotics in the intervertebral disc. Spine (Phila Pa 1976) 1994;19:2619-25.

16. Rimoldi RL, Haye W. The use of antibiotics for wound prophylaxis in spinal surgery. Orthop Clin North Am 1996;27:47-52.

17. Piotrowski WP, Krombholz MA, Muhl B. Spondylodiscitis after lumbar disk surgery. Neurosurg Rev 1994;17:189-93.

18. Rohde V, Meyer B, Schaller C, Hassler WE. Spon- dylodiscitis after lumbar discectomy. Incidence and a proposal for prophylaxis. Spine (Phila Pa 1976) 1998;23:615-20.

19. Rubinstein E, Findler G, Amit P, Shaked I. Perioperative prophylactic cephazolin in spinal surgery: a double-blind placebo-controlled trial. J Bone Joint Surg Br 1994;76:99-102.

20. Fraser RD, Osti OL, Vernon-Roberts B. Iatrogenic discitis: the role of intravenous antibiotics in prevention and treatment: an experimental study. Spine (Phila Pa 1976) 1989;14:1025-32.

21. Boscardin JB, Ringus JC, Feingold DJ, Ruda SC. Human intradiscal levels with cefazolin. Spine (Phila $\mathrm{Pa}$ 1976) 1992;17:S145-8.

22. Rhoten RL, Murphy MA, Kalfas IH, Hahn JF, Washington JA. Antibiotic penetration into cervical discs. Neurosurgery 1995;37:418-21.

23. Scuderi GJ, Greenberg SS, Banovac K, Martinez OV, Eismont FJ. Penetration of glycopeptide antibiotics in nucleus pulposus. Spine (Phila Pa 1976) 1993;18:2039-42.

24. Lang R, Folman Y, Ravid M, Bental T, Gepstein R. Sequential levels of ceftriaxone in intervertebral disc removed as part of scoliosis surgery. Clin Orthop Relat Res 1995;(315):209-11.

25. Hoelscher GL, Gruber HE, Coldham G, Grigsby JH, Hanley EN Jr. Effects of very high antibiotic concentrations on human intervertebral disc cell proliferation, viability, and metabolism in vitro. Spine (Phila Pa 1976) 2000;25:1871-7.

26. Gibson MJ, Karpinski MR, Slack RC, Cowlishaw WA, Webb JK. The penetration of antibiotics into the normal intervertebral disc. J Bone Joint Surg $\mathrm{Br}$ 1987;69:784-6.

27. Classen DC, Evans RS, Pestotnik SL, Horn SD, Menlove RL, Burke JP. The timing of prophylactic administration of antibiotics and the risk of surgical-wound infection. N Engl J Med 1992;326:281-6.

28. Guiboux JP, Cantor JB, Small SD, Zervos M, Herkowitz HN. The effect of prophylactic antibiotics on iatrogenic intervertebral disc infections. a rabbit model. Spine (Phila Pa 1976) 1995;20:685-8.

29. Capen DA, Calderone RR, Green A. Perioperative risk factors for wound infections after lower back fusions. Orthop Clin North Am 1996;27:83-6.

30. Quartey GR, Polyzoidis K. Intraoperative antibiotic prophylaxis in neurosurgery: a clinical study. Neurosurgery 1981;8:669-71. 